\title{
Lysine Biosynthesis in Bacteria: A Metallodesuccinylase as a Potential Antimicrobial Target
}

\author{
Danuta M. Gillner \\ Silesian University of Technology \\ Daniel P. Becker Ph.D. \\ Loyola University Chicago, dbecke3@luc.edu \\ Richard C. Holz \\ Loyola University Chicago, rholz1@luc.edu
}

Follow this and additional works at: https://ecommons.luc.edu/chemistry_facpubs

Part of the Biochemistry Commons, Chemistry Commons, and the Microbiology Commons

Author Manuscript

This is a pre-publication author manuscript of the final, published article.

\section{Recommended Citation}

Gillner, Danuta M.; Becker, Daniel P. Ph.D.; and Holz, Richard C.. Lysine Biosynthesis in Bacteria: A Metallodesuccinylase as a Potential Antimicrobial Target. JBIC Journal of Biological Inorganic Chemistry, 18, 2: 155-163, 2013. Retrieved from Loyola eCommons, Chemistry: Faculty Publications and Other Works, http://dx.doi.org/10.1007/s00775-012-0965-1

This Article is brought to you for free and open access by the Faculty Publications and Other Works by Department at Loyola eCommons. It has been accepted for inclusion in Chemistry: Faculty Publications and Other Works by an authorized administrator of Loyola eCommons. For more information, please contact ecommons@luc.edu. c) (†) $\ominus$

This work is licensed under a Creative Commons Attribution-Noncommercial-No Derivative Works 3.0 License. (c) 2012 SBIC 


\title{
Lysine Biosynthesis in Bacteria:
}

\section{A Metallodesuccinylase as a Potential Antimicrobial Target}

\author{
Danuta M. Gillner ${ }^{1,2 *}$, Daniel Becker ${ }^{1}$, and Richard C. Holz ${ }^{1 *}$
}

Contribution from the Department of Chemistry and Biochemistry, Loyola University-

Chicago, 1068 W. Sheridan Rd., Chicago, IL 60626 and the Department of Chemistry,

Silesian University of Technology, ul. Krzywoustego 4, 44-100 Gliwice, Poland

Running Title: dapE-encoded $N$-succinyl-L,L-diaminopimelic acid desuccinylase

${ }^{1}$ Loyola University Chicago, ${ }^{2}$ Silesian University of Technology

†This work was supported by the National Institutes of Health (R15 AI085559-01A1, RCH).

*Address correspondence to: Richard C. Holz, Department of Chemistry, Loyola UniversityChicago, 1068 W. Sheridan Rd., Chicago, IL 60626, Phone (773) 508-3092, Fax: (773) 5083086, Internet: rholz1@luc.edu or Danuta M. Gillner, Department of Chemistry, Silesian University of Technology, ul. Krzywoustego 4, 44-100 Gliwice, Poland, Phone +48 3223727 91, Fax: +48 3223710 32, Internet: Danuta.Gilner@polsl.pl.

\section{Antibiotic Resistance}




\section{Abstract}

In this review, we summarize the recent literature on the dapE-encoded $N$-succinylL,L-diaminopimelic acid desuccinylase (DapE) enzymes with an emphasis on structurefunction studies, which have provided insight into the catalytic mechanism of DapE enzymes. Crystallographic data has also provided insight into residues that might be involved in substrate, and hence inhibitor recognition and binding. These data have led to the design and synthesis of several new DapE inhibitors, which are described along with what is known about how inhibitors interact with the active site of DapE enzymes including the efficacy of a moderately strong DapE inhibitor.

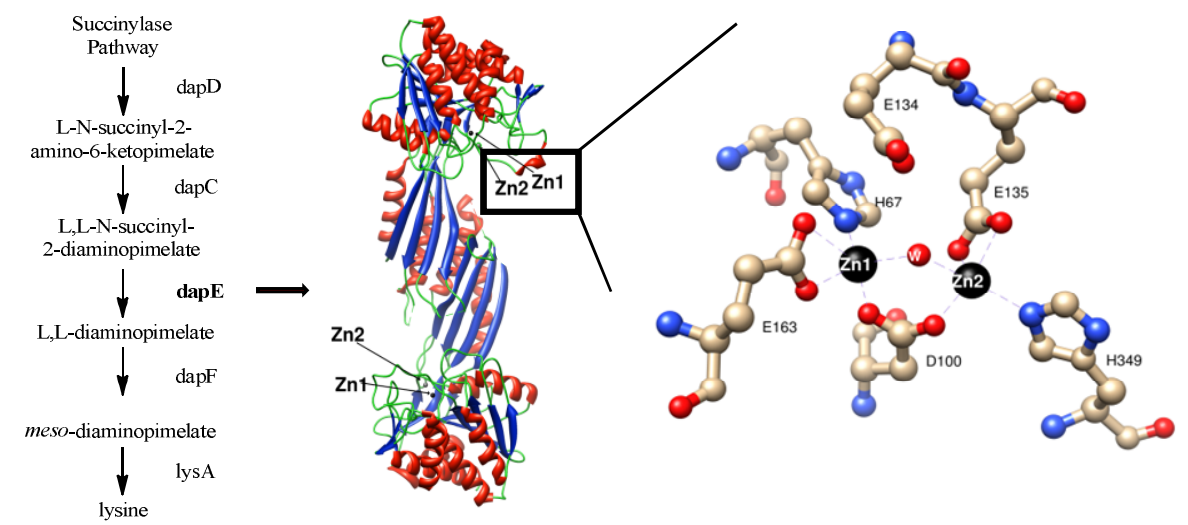

Key Words: DapE, Metallohydrolase, Antibiotics, Zinc, X-ray Crystallography, Inhibitor Design, Catalytic Mechanism. 
The emergence of antibiotic-resistant bacterial infections has created a significant and growing medical problem in the United States and throughout the world [1-5]. Antibiotic resistance has been recognized since the introduction of penicillin more than 50 years ago when penicillin-resistant infections caused by Staphylococcus aureus rapidly appeared [3, 6]. Because bacteria have been exposed to many of the currently available antibiotics such as $\beta-$ lactams, fluoroquinolones, macrolides, tetracyclines, aminoglycosides, glycopeptides, or trimethoprim combinations for years, they have evolved resistance to these drugs due to mutation or the acquisition of genes that impart resistance from other organisms [3, 7-10]. In fact, several pathogenic bacteria, some of which were thought to have been eradicated, have made a significant resurgence due to bacterial resistance to antibiotics $[3,6]$. For example, tuberculosis is currently the leading cause of death in adults by an infectious disease worldwide, which is significant given that death rates due to tuberculosis had declined to near imperceptible levels in industrial nations [11-13]. According to the CDC, several bacterial strains currently exhibit multidrug resistance with more than $60 \%$ hospital acquired infections in the United States caused by the so-called ESKAPE pathogens (Enterococcus faecium,

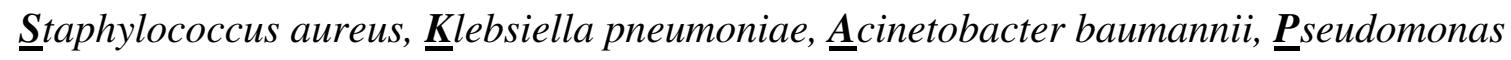

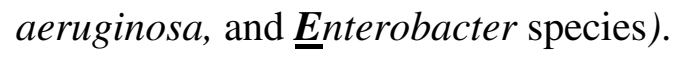

Antibiotics work by interfering with a vital bacterial cell function at a specific cellular target by either killing the bacteria or arresting their multiplication [5]. This allows the patient's immune system to clear the bacteria from the body. Inhibitors of cell wall biosynthesis (vancomycin and $\beta$-lactams, to name a few) have proven to be very potent antibiotics, evidence that interfering with cell-wall synthesis has deleterious effects on bacterial cell survival. Enzymes that are targeted by these antibiotics tend to be present in all bacteria and are highly similar in structure and function, such that certain antibiotics kill or inhibit the growth of a broad range of bacterial species (i.e., broad-spectrum antibiotics) [3, 7- 
10]. Unfortunately, only two new classes of anti-bacterial drugs have emerged since 1962. According to the Infectious Diseases Society of America at least ten new systemic antibacterial drugs should enter the market by the year 2020; however, most of these are derivatives of existing classes of antibiotics. Since every antibiotic has a finite lifetime, as resistance will ultimately occur particularly if the same enzymes are repeatedly targeted, development of new classes of inhibitors that target previously untargeted cellular enzymes is essential to retain control of infectious disease $[14,15]$.

\section{Lysine Biosynthetic Pathway}

Based on bacterial genetic information, the meso-diaminopimelate ( $m \mathrm{DAP}) / \mathrm{lysine}$ biosynthetic pathway offers several potential antibacterial enzyme targets that have yet to be explored (Figure 1) [16-18]. One of the products of this pathway, lysine, is required in protein synthesis and is also used in the peptidoglycan layer of Gram-positive bacterial cell walls. A second product of this pathway, $m \mathrm{DAP}$ is an essential component of the peptidoglycan cell wall for Gram-negative bacteria, providing a link between polysaccharide strands. Since lysine is an essential amino acid and is not synthesized by humans, it must be ingested. However, most bacteria, plants and algae synthesize lysine and $m \mathrm{DAP}$ from aspartic acid through three related pathways that diverge after the production of Ltetrahydrodipicolinate $[16,17,19]$. The presence of multiple biosynthetic pathways in bacteria for the synthesis of $m \mathrm{DAP} / \mathrm{lysine}$ highlights the importance of $m \mathrm{DAP} / \mathrm{lysine}$ for bacterial cell survival.

The succinylase pathway is the primary biosynthetic pathway for $m \mathrm{DAP} / \mathrm{lysine}$ and is utilized by all Gram-negative and most Gram-positive bacteria [16]. The dehydrogenase pathway forms $m$ DAP directly from L-tetrahydrodipicolinate but this is a high-energy transformation and is limited to only a few Bacillus species [16]. The acetylase pathway is 
also a minor biosynthetic pathway for $m \mathrm{DAP}$ production and is also limited to only a few Bacillus species [17]. One of the enzymes in the succinylase pathway, the dapE-encoded $N$ succinyl-L,L-diaminopimelic acid desuccinylase (DapE), is a $\mathrm{Zn}(\mathrm{II})$ containing metallohydrolase. It has been shown that deletion of the gene encoding for DapE is lethal to Helicobacter pylori and Mycobacterium smegmatis [20,21]. Even in the presence of lysine supplemented media H. pylori was unable to grow, suggesting that lysine can't be synthesized by other pathways or imported. Therefore, DapE's appear to be essential for cell growth and proliferation and are part of a biosynthetic pathway that is the only source of lysine in most bacteria. Since there are no similar biosynthetic pathways in mammals, DapE's appear to be potential targets for inhibitors that may possess antimicrobial activity [16].

\section{DapE's Contain a Dinuclear Zn(II) Active Site}

DapE's catalyze the hydrolysis of $N$-succinyl-L,L-diaminopimelic acid (L,L-SDAP), forming L,L-diaminopimelic acid and succinate (Figure 2) [19]. DapE's have been purified from multiple sources and genes identified in a large number of pathogenic Gram-positive and Gram-negative bacteria including all of the ESKAPE pathogens [20-25]. Alignment of several of the DapE gene sequences show a minimum of $49 \%$ identity [26]. Significantly, all of the residues that are metal binding ligands in the M28 family of dinuclear $\mathrm{Zn}$ (II)-dependent metalloproteases [27], including the aminopeptidase from Vibrio proteolyticus (Aeromonas proteolytica, AAP) and the carboxypeptidase from Pseudomonas sp strain-RS-16 (CPG2) [28, 29] are strictly conserved in all DapE enzymes. Since the catalytic activity of DapE's require Zn(II) and both CPG2 and AAP possess ( $\mu$-aquo)( $\mu$-carboxylato)dizinc(II) active sites with one terminal carboxylate and one histidine residue at each metal site, a similar active site was proposed for DapE enzymes [19, 30-32]. Evidence for a dinuclear Zn(II)-active site in DapE enzymes was obtained via Zn K-edge Extended X-ray Absorption Fine Structure (EXAFS) spectra of the DapE from H. influenzae in the presence of one and two equivalents of $\mathrm{Zn}(\mathrm{II})$ 
(i.e. [Zn_(DapE)] and [ZnZn(DapE)]) [33]. Fourier transforms of the Zn EXAFS are dominated by a peak at $c a .2 .0 \AA$, which was best fit assuming $c a .5(\mathrm{~N}, \mathrm{O})$ scatterers at 1.96 and $1.98 \AA$ for $\left[\mathrm{Zn} \_(\mathrm{DapE})\right]$ and $[\mathrm{ZnZn}(\operatorname{DapE})]$, respectively. Inclusion of a sulfur (S) atom provided poorer fits based on Debye-Waller Factors. A second-shell feature at ca. $3.34 \AA$ appears in the $[\mathrm{ZnZn}(\mathrm{DapE})]$ EXAFS spectrum but is significantly diminished in [Zn_(DapE)]. These data confirmed that DapE enzymes can bind two Zn(II) ions that form a dinuclear site.

Both AAP and CPG2 contain active site His residues that function as ligands to the $\mathrm{Zn} 1$ and $\mathrm{Zn} 2$ centers. Based on sequence alignment of the DapE from $H$. influenzae with AAP and CPG2, both $\mathrm{H} 67$ and H349 were predicted to be $\mathrm{Zn}(\mathrm{II})$ ligands [32]. In an effort to clearly define the active site residues and provide insight into the structural properties of each divalent metal ion in DapE enzymes, the H67A and H349A DapE mutant enzymes were prepared. The H67A DapE enzyme exhibited a decrease in catalytic efficiency ( 180-fold) compared to WT DapE towards the substrate L,L-SDAP. No catalytic activity was observed for H349A under the experimental conditions used. EPR and UV-Vis data indicated that the Co(II) ion bound to H349A-DapE is analogous to that of WT DapE after the addition of a single $\mathrm{Co}(\mathrm{II})$ ion. The addition of one equivalent of $\mathrm{Co}(\mathrm{II})$ to H67A DapE provided spectra that are very different from the first Co(II) binding site of the WT enzyme, but similar to the second binding site. The UV-Vis and EPR data, in conjunction with the kinetic data, are consistent with the assignment of H67 and H349 as active site metal ligands for DapE. Furthermore, these data suggest that H67 is a ligand in the first metal binding site while H349 resides in the second metal binding site.

Sequence alignment with AAP and CPG2 also suggested that the active site residue in the DapE from H. influenzae, E134, likely functioned as the general acid/base during the hydrolysis reaction catalyzed by DapE [19]. In order to elucidate the catalytic role of E134, the E134A and E134D mutant DapE enzymes were prepared [34]. The Michaelis constant 
$\left(K_{\mathrm{m}}\right)$ was found to not change upon substitution to aspartate but the $k_{\text {cat }}$ values changed drastically in the order: glutamate $\left(140 \mathrm{~s}^{-1}\right)$, aspartate $\left(0.13 \mathrm{~s}^{-1}\right)$, and alanine (none detected). Examination of the $\mathrm{pH}$ dependence of the kinetic constants $k_{\text {cat }}$ and $K_{\mathrm{m}}$ for the E134D enzyme revealed ionizations at $\mathrm{pH} 6.4,7.4$, and $~ 9.7$. Isothermal titration calorimetry (ITC) studies on WT DapE provided $K_{\mathrm{d}}$ values for the first and second $\mathrm{Zn}(\mathrm{II})$ binding sites of 4.4 and 13.6 $\mu \mathrm{M}$. ITC experiments on E134D- and E134A-DapE revealed a significant weakening in metal $K_{\mathrm{d}}$ values of 2.9 and 1.4 times for the first metal binding event, respectively. Interestingly, UV-Vis and EPR spectra obtained on Co(II)-substituted E134D and E134A DapE did not reveal any significant changes suggesting that both Co(II) ions reside in distorted trigonal bipyramidal coordination geometries [35]. Combination of these data indicates that E134 is intrinsically involved in the hydrolysis reaction catalyzed by DapE and likely plays the role of a general acid/base.

\section{Structural Characterization of DapE Enzymes}

A major limitation in developing a previously undescribed class of antimicrobials that target DapE enzymes was the lack of knowledge about their active site structure including potential residues involved in substrate binding. The X-ray crystal structure of the DapE enzyme from $N$. meningitidis was reported at $1.9 \AA$ but was in the apo-form, providing little information about the $\mathrm{Zn}$ (II) active site [36]. Recently, the X-ray crystal structure of the DapE from $H$. influenzae was reported for both the mono and dinuclear $\mathrm{Zn}$ (II) forms at 2.0 and 2.3 A resolution, respectively [37]. Similar to the DapE from N. meningitidis, the DapE from $H$. influenzae forms a homodimer where the catalytic domain consists of an $\alpha / \beta$ globular domain with a twisted $\beta$-sheet hydrophobic core sandwiched between $\alpha$-helices (Figure 3 ). The active-site cleft is located in the center of the catalytic domain above the centrally located 
parallel strands of the $\beta$-sheet and is covered by loops. The location and the architecture of the active site are strikingly similar to the active sites of CPG2 and AAP. ${ }^{28,29}$

The active site of the mononuclear $\mathrm{Zn}(\mathrm{II})$ form of DapE ([Zn_(DapE)]), reveals that Zn1 resides in a distorted tetrahedral geometry coordinated by the carboxylate oxygens (OD1) of D100 and (OE1) of E163 as well as one nitrogen atom (NE2) of H67 (Figure 4). The remaining coordination site is filled by an oxygen atom provided from a water molecule. In the dinuclear $\mathrm{Zn}(\mathrm{II})$ form of $\mathrm{DapE}$ ([ZnZn(DapE)]), the active site contains two $\mathrm{Zn}$ (II) ions at a distance of $3.36 \AA$ compared with $3.45 \AA$ for AAP and $3.25 \AA$ for $\mathrm{CPG}_{2}{ }^{28,29}$ Each of the $\mathrm{Zn}(\mathrm{II})$ ions adopts a distorted tetrahedral geometry and is coordinated by one imidazole group (H67 for Zn1 and H349 for Zn2) and one carboxylate group (E163 for Zn1 and E135 for Zn2). Both $\mathrm{Zn}(\mathrm{II})$ ions are bridged by an additional carboxylate group (D 100) on one side and water/hydroxide on the opposite side, forming a ( $\mu$-aquo)( $\mu$-carboxylato)dizinc(II) core with one terminal carboxylate and one histidine residue at each metal site. These structures confirm the assignment of H67 and H349 as active site ligands with H67 residing in the first metal binding site as well as the role of Glu134.

Inspection of the X-ray crystal structures of [Zn_(DapE)] and[ZnZn(DapE)], combined with surface analysis, revealed a crescent-shaped cavity that extends along the catalytic domain and surrounds the active site (Figure 5). This well-defined, negatively charged cavity is shaped from the top by strand $\beta 12$ and $\alpha 8$ and in the middle by the loop connecting these two elements. The bottom of the cavity is formed by a loop connecting $\beta$ strands $\beta 6$ and $\beta 7$, and a loop connecting $\beta 5$ and $\alpha 4$. Taking into account the linear character of the substrate, it is likely that the substrate binds in an extended conformation [38], lining up along the grove with the peptide bond positioned over the active site metals. It is conceivable that substrate binding is further stabilized by interaction of the substrate carboxylate groups with positively charged amino acid side chains. Potential candidates include K175, R258, and 
R329 (Figure 6). In the [ZnZn(DapE)] structure, R258, and R329 form a charged dipole interaction with a sulfate ion, a possible mimic of the carboxylic group of the substrate. R329 is centrally positioned in a positively charged pocket that it forms together with R258. Interestingly, the active site pocket of CPG2 contains an arginine that is conserved in DapE (R329) and has been proposed to bind the carboxylate group of the side chain. These data, in combination with previously reported inhibitor binding studies [32, 33, 39, 40], indicate that DapE represents an excellent target for a highly specific drug that should have high efficacy and low toxicity.

\section{Proposed Catalytic Mechanism of DapE}

The X-ray structures of [Zn_DapE] and [ZnZn_DapE] provide a structural foundation for a proposed reaction mechanism of DapE [19, 32, 34]. Analysis of the available structures along with the previously reported kinetic and spectroscopic data for DapE enzymes allowed a detailed mechanism of catalysis for DapE's to be proposed (Figure 7) [37]. Based on the proposed catalytic mechanism for AAP [41, 42], the first step in catalysis for DapE's is likely recognition of the L,L-SDAP side chain by the crescent-shaped cavity adjacent to the Zn1 site. Next, the peptide carbonyl oxygen of L,L-SDAP coordinates to Zn1 and expands its coordination number from four to five, activating the carbonyl for nucleophilic attack.

Deprotonation of the metal-bound water molecule by E134 to form a nucleophilic hydroxide moiety is consistent with the postulated $\mathrm{p} K_{\mathrm{a}}$ of the zinc-bound water molecule [19]. Once the zinc-bound hydroxide is formed, it can attack the activated carbonyl carbon of the substrate,

forming an -1- $\mu$-transition-state complex [33]. Solvent kinetic isotope effect studies yielded an inverse isotope effect that was explained by the attack of a zinc-bound hydroxide on the amide carbonyl [19]. E134 may provide a proton to the penultimate amino nitrogen, similar to that observed for AAP, returning it to its ionized state thus facilitating product release. Once the products are released, a water molecule bridging the two metal ions is replaced. In 
the absence of a second metal ion, the catalytic mechanism does not likely change markedly as $\mathrm{H} 349$ is in position to assist in orienting the substrate properly in the active site through the formation of a hydrogen bond with a carboxylate side chain of the substrate, thereby stabilizing the transition-state intermediate reminiscent to proposals for the mono-metalated forms of AAP and the methionine aminopeptidase from E. coli [43-45]. In the presence of a dinuclear site, the second metal ion likely coordinates either the peptide carbonyl oxygen in a bridging fashion or a carboxylate side chain of the substrate.

\section{Design and Synthesis of DapE Inhibitors}

The design and synthesis of novel DapE inhibitors requires information regarding substrate specificity. Therefore, the four isomers of SDAP as well as a number of acetylated amino acids have been examined as potential substrates. DapE was not able to hydrolyze the D,D-, L,D-, or D,L-isoforms of SDAP confirming that L,L-SDAP is the only known biological substrate for DapE enzymes. Moreover, no hydrolytic degradation was observed for any acetylated amino acids tested [25, 32], therefore, the DapE active site has strict substrate specificity with regard to both functional groups and stereochemistry. These data also suggest that the carboxylate of the succinyl moiety forms an important interaction within the active site of DapE since the acetylated amino acids could not be hydrolyzed.

Alternatively, the acetylated versions of these amino acids may introduce a repulsive interaction because of bulky alkyl groups, thus, preventing hydrolysis. Given the lack of hydrolytic activity towards the SDAP isoforms, a series of potential DapE inhibitors based on SDAP bearing different $N$-linked acyl side chains terminated with (i) a carboxyl group or (ii) a lipophilic moiety, were synthesized. Unfortunately, none of these compounds functioned as potent inhibitors of DapE [32, 33, 39, 40]. Finally, D,L-succinyl aminopimelate was examined, which only differs from the natural substrate, L,L-SDAP, by the absence of the 
amine group on the amino acid side chain. Interestingly, DapE could not hydrolyze this compound implying that the amine provides an important interaction for substrate binding.

The next step in inhibitor design for DapE enzymes involved understanding how inhibitors bind to the active site. To date, no X-ray structural data for a DapE enzyme in the presence of an inhibitor has been reported; however, Zn K-edge EXAFS spectra of $[\mathrm{ZnZn}(\mathrm{DapE})]$ in the presence of the competitive inhibitors 2- carboxyethylphosphonic acid $\left(\mathrm{CEPA} ; K_{\mathrm{i}}=800 \mu \mathrm{M}\right)$ and 5- mercaptopentanoic acid (MSPA; $\left.K_{\mathrm{i}}=6 \mu \mathrm{M}\right)$ have been reported.[33] Phosphonic acid-containing compounds have been shown to be potent inhibitors of metallohydrolases, including AAP and the bovine lens leucine aminopeptidase (blLAP) and have been used as probes of the transition-state of hydrolysis reactions. The EXAFS data for [ZnZn(DapE)]-CEPA indicate that the average coordination number of each

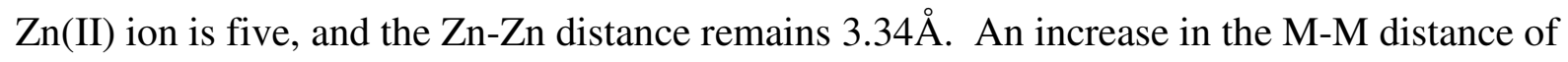
[ZnZn(AAP)], from 3.5 to $3.9 \AA$, is observed upon the addition of the transition-state analog inhibitor L-leucine- phosphonic acid (LPA), which contains a similar ligating group to CEPA [46]. The X-ray crystal structure and EXAFS data for [ZnZn(AAP)]-LPA, reveal that the bridging water molecule is displaced by LPA resulting in an $\eta-1,2-\mu-$ phosphonate bridge and an increase in the $\mathrm{Zn}-\mathrm{Zn}$ distance of $0.4 \AA$. The fact that the $\mathrm{Zn}-\mathrm{Zn}$ distance is not altered upon CEPA binding to [ZnZn(DapE)] suggests an $\eta-1-\mu$-phosphonate bridge exists, similar to the binding mode of LPA to blLAP $[46,47]$. On the other hand, MSPA binding to $[\mathrm{ZnZn}(\mathrm{DapE})]$ has a marked effect on the $\mathrm{Zn} \mathrm{K}$-edge, suggesting that the average electronic environment of the dinuclear $\mathrm{Zn}$ (II) site has changed significantly. The observed shift to lower energy in the absorption edge position is indicative of a net increase in electron density at the dinuclear $\mathrm{Zn}$ (II) site, consistent with a sulfur ligand. In addition, the EXAFS data for [ZnZn(DapE)]-MSPA reveals a new feature at $2.3 \AA$ that is highly characteristic of a direct zinc-sulfur interaction. Moreover, the M-M distance is lengthened from $3.34 \AA$ to $3.64 \AA$. 
These data indicate that the thiol group of MSPA binds to one or both of the $\mathrm{Zn}$ (II) ions in the active site of DapE.

The fact that thiol based molecules were shown to be moderately strong inhibitors of DapE provided insight into examining bifunctional molecules that contained, in addition to a thiol zinc binding group (ZBG), a carboxylate moiety that could interact with the positivelycharged lysine and arginine side chains that purportedly reside near the active site. These studies led to the identification of several low micromolar inhibitors of DapE, all of which, like MSPA, contain a thiol functional group [39]. Thiol containing compounds are typically good inhibitors of $\mathrm{Zn}$ (II) metalloproteins since $\mathrm{Zn}$ (II) is a soft acid and thiols are soft bases [48]. One of the better inhibitors was L-penicillamine (Figure 8) which exhibited an IC50 value of $13.7 \mu \mathrm{M}$, and a measured $K_{\mathrm{i}}$ of $4.6 \mu \mathrm{M}$ (competitive). DapE is stereoselective with respect to recognition of inhibitors, as D-penicillamine gave an $\mathrm{IC}_{50}$ of $50 \mu \mathrm{M}$. Given the success with these carboxylic acid-containing thiols, L-captopril (Figure 8) an angiotensinconverting enzyme (ACE) inhibitor was examined, which contains the requisite $\mathrm{ZBG}$ and carboxylate functionalities. L-Captopril exhibited an $\mathrm{IC}_{50}$ of $3.3 \mu \mathrm{M}$ and a measured $K_{\mathrm{i}}$ of 1.8 $\mu \mathrm{M}$ (competitive). Again, the binding is stereoselective, as D-captopril was an order of magnitude less potent, with an $\mathrm{IC}_{50}$ of $42.0 \mu \mathrm{M}$. Given the good potency of L-captopril, another ACE inhibitor, enalapril, was screened but did not show any potency towards DapE. 4-Mercaptobutyric acid is also an inhibitor of DapE and exhibits an IC50 value of $43 \mu \mathrm{M}$ while 2-mercaptobenzoic acid has a measured IC50 value of $34 \mu \mathrm{M}$. For these low micromolar inhibitors, it was hypothesized that the presence of a negatively-charged carboxylate in the molecule participates in an ionic interaction with a positively-charged lysine or arginine near the active site.

Given that L-captopril functions as a moderately strong inhibitor of DapE, its efficacy was examined using a standard plate assay [39]. Application of L-captopril or L- 
penicillamine directly to plates cultured with $E$. coli showed a dose-responsive antibiotic activity for L-captopril. Very little inhibition is observed for $1 \mathrm{mg}$ of L-captopril, but $5 \mathrm{mg}$ and $20 \mathrm{mg}$ demonstrate a clear positive antibiotic result. These data suggest that L-captopril can cross the bacterial cell membrane and function to inhibit bacterial cell growth. Whether DapE is the cellular target of L-captopril was not addressed, however, a recent study suggested that DapE is not the main target of L-captopril antimicrobial activity since Lcaptopril inhibited S. enterica and E. coli in a DapE-independent manner [49]. Clearly, further studies are needed to verify DapE as an antimicrobial target and such studies will necessitate the discovery of strong binding inhibitors that are specific for DapE enzymes.

\section{Concluding Remarks}

Bacterial infections, some of which were thought to be eradicated, have made a significant resurgence due to bacterial resistance to all known antibiotics [2-4]. Consequently, new compounds that combat these pathogens and target enzymes involved in bacterial cell wall synthesis or pathways involved in cell replication are in high demand [5057]. That the meso-diaminopimelate $(m \mathrm{DAP}) /$ lysine biosynthetic pathway offers several potential anti-bacterial targets that have yet to be explored. While our understanding of the catalytic mechanism of DapE has markedly improved over the past few years, it has not yet been entirely delineated. For example, several substrate binding steps have been proposed and the roles of each metal ion during catalysis are still in question. Therefore, more experimental evidence is needed to distinguish between the competing mechanistic proposals for DapE. Even so, results obtained from the studies described in this review have provided new insight into the structure and function of DapE enzymes and have led to new medicinal chemistry leads. Additional insight into the catalytic mechanism of DapE and the 
determinants of substrate-binding will be critical for the rational design of future selective DapE inhibitors that may function as a new class of antimicrobial agents. 


\section{References}

1. Prevention CfDCa (1995) MMWR Morb. Mortal. Wkly Rep. 44:1-13

2. Howe RA, Bowker KE, Walsh TR, Feest TG, MacGowan AP (1998) Lancet 351:601602

3. Levy SB (1998) Sci. Am. 278:46-53

4. Chin J (1996) New Scientist 152:32-35

5. Henery CM (2000) C\&E News 78:41-58

6. Nemecek S (1997) Sci. Am. 276:38-39

7. Miller JB (2000)The Pharmaceutical-Century: Ten Decades of Drug Discovery; Supplement to ACS: Washington DC, pp. 52-71

8. Lesney MS, Frey R (2000)The Pharmaceutical-Century: Ten Decades of Drug Discovery; Supplement to ACS: Washington DC, pp. 110-129

9. Frey R, Lesney MS (2000)The Pharmaceutical-Century: Ten Decades of Drug Discovery; Supplement to ACS: Washington DC, pp. 92-109

10. Tweedy BD, Lesney MS (2000)The Pharmaceutical-Century: Ten Decades of Drug Discovery; Supplement to ACS: Washington DC, pp. 72-91

11. Snider DE, Raviglione M, Kochi A (1994) In: Bloom BR (ed) Global burden of tuberculosis. ASM Press, Washington DC, pp. 3-11

12. Dolin PJ, Raviglione MC, Kochi A (1994) Bull. W. H. O. 72:213-220

13. Raviglione MC, Snider DE, Kochi A (1995) JAMA 273:220-226

14. Teuber M (1999) Cell. Mol. Life Sci. 56:755-763

15. Miller JR, Dunham S, Mochalkin I, Banotai C, Bowman M, Buist S, Dunkle B, Hanna D, Harwood HJ, Huband MD, Karnovsky A, Kuhn M, Limberakis C, Liu JY, Mehrens S, Mueller WT, Narasimhan L, Ogden A, Ohren J, Prasad JV, Shelly JA, Skerlos L, Sulavik M, Thomas VH, VanderRoest S, Wang L, Wang Z, Whitton A, Zhu T, Stover CK (2009) Proc Natl Acad Sci U S A. 106:1737-1742 DOI 10.1073/pnas.0811275106

16. Scapin G, Blanchard JS (1998) Adv. Enzymol. 72:279-325

17. Born TL, Blanchard JS (1999) Cur. Opin. Chem. Biol. 3:607-613 
18. Girodeau J-M, Agouridas C, Masson M, R. P, LeGoffic F (1986) J. Med. Chem. 29:1023-1030

19. Born TL, Zheng R, Blanchard JS (1998) Biochemistry 37:10478-10487

20. Karita M, Etterbeek ML, Forsyth MH, Tummuru MR, Blaser MJ (1997) Infect. Immun. 65:4158-4164

21. Pavelka MS, Jacobs WR (1996) J. Bacteriol. 178:6496-6507

22. Bouvier J, Richaud C, Higgins W, Bögler O, Stragier P (1992) J. Bacteriol. 174:52655271

23. Fuchs TM, Schneider B, Krumbach K, Eggeling L, Gross R (2000) J. Bacteriol. $182: 3626-3631$

24. Shaw-Reid CA, McCormick MM, Sinskey AJ, Stephanopoulos G (1999) Appl. Microbiol. Biotechnol. 51:325-333

25. Lin Y, Myhrman R, Schrag ML, Gelb MH (1988) J. Biol. Chem. 263:1622-1627

26. Gillner DM, Bienvenue DL, Nocek BP, Joachimiak A, Zachary V, Bennett B, Holz RC (2009) J. Biol. Inorg. Chem. 14:1-10

27. Barrett AJ, Rawlings ND, Woessner JF (eds) (1998) Handbook of Proteolytic Enzymes. Academic Press, London

28. Rowsell S, Pauptit RA, Tucker AD, Melton RG, Blow DM, Brick P (1997) Structure $5: 337-347$

29. Desmarais W, Bienvenue DL, Bzymek KP, Petsko GA, Ringe D, Holz RC (2006) J. Biol. Inorg. Chem. 11:398-408

30. Chevrier B, Schalk C, D'Orchymont H, Rondeau J-M, Moras D, Tarnus C (1994) Structure 2:283-291

31. Greenblatt HM, Almog O, Maras B, Spungin-Bialik A, Barra D, Blumberg S, Shoham G (1997) J. Mol. Biol. 265:620-636

32. Bienvenue DL, Gilner DM, Davis RS, Bennett B, Holz RC (2003) Biochemistry 42:10756-10763

33. Cosper NJ, Bienvenue DL, Shokes JE, Gilner DM, Tsukamoto T, Scott R, Holz RC (2003) J. Am. Chem. Soc. 125:14654-14655 
34. Davis R, Bienvenue D, Swierczek SI, Gilner DM, Rajagopal L, Bennett B, Holz RC (2006) J. Biol. Inorg. Chem. 11:206-216

35. Bennett B (2010) In: Hanson G, Berliner L (eds) Biol. Magn. Reson., Metals in Biology. Springer, New York, pp. 345-370

36. Badger J, Sauder JM, Adams JM, Antonysamy S, Bain K, Bergseid MG, Buchanan SG, Buchanan MD, Batiyenko Y, Christopher JA, Emtage S, Eroshkina A, Feil I, Furlong EB, Gajiwala KS, Gao X, He D, Hendle J, Huber A, Hoda K, Kearins P, Kissinger C, Laubert B, Lewis HA, Lin J, Loomis K, Lorimer D, Louie G, Maletic M, Marsh CD, Miller I, Molinari J, Muller-Dieckmann HJ, Newman JM, Noland BW, Pagarigan B, Park F, Peat TS, Post KW, Radojicic S, Ramos A, Romero R, Rutter ME, Sanderson WE, Schwinn KD, Tresser J, Winhoven J, Wright TA, Wu L, Xu J, Harris TJ (2005) Proteins 60:787-796

37. Nocek BP, Gillner DM, Fan Y, Holz RC, Joachimiak A (2010) J. Mol. Biol. 397:617626 DOI 10.1016/j.jmb.2010.01.062

38. Tyndall JDA, Nall T, Fairlie DP (2005) Chem. Rev. 105:973-999

39. Gillner DM, Armush N, Holz RC, Becker D (2009) Bioorg. Med. Chem. Lett. $19: 6350-6352$

40. Vaněk V, Pícha J, Buděšínský M, Šanda M, Jiráček J, Holz RC, Hlaváček J (2010) Protein Pept. Lett. 17:405-409

41. Ustynyuk L, Bennett B, Edwards T, Holz RC (1999) Biochemistry 38:11433-11439

42. Stamper CC, Bienvenue DL, Moulin A, Bennett B, Ringe D, Petsko GA, Holz RC (2004) Biochemistry 43:9620-9628

43. Copik AJ, Swierczek SI, Lowther WT, D'souza VM, Matthews BW, Holz RC (2003) Biochemistry 42:6283-6292

44. Ye QZ, Xie SX, Ma ZQ, Huang M, Hanzlik RP (2006) Proc Natl Acad Sci U S A. 103:9470-9475

45. Holz RC (2002) Coord. Chem. Rev 232:5-26

46. Stamper CC, Bennett B, Edwards T, Holz RC, Ringe D, Petsko GA (2001) Biochemistry 40:7035-7046 
47. Sträter N, Lipscomb WN (1995) Biochemisty 34:9200-9210

48. Jacobsen FE, Lewis JA, Cohen SM (2007) ChemMedChem 2:152-171

49. Uda NR, Creus M (2011) Bioinorganic Chemistry and Applications 2011:6 DOI $10.1155 / 2011 / 306465$

50. Arfin SM, Kendall RL, Hall L, Weaver LH, Stewart AE, Matthews BW, Bradshaw RA (1995) Proc. Natl. Acad. Sci. USA 92:7714-7718

51. Ben-Bassat A, Bauer AK, Chang S-Y, Myambo K, Boosman A, Chang S (1987) J. Bacteriol. 169:751-757

52. Ben-Bassat A, Bauer K (1987) Nature 326:315

53. Chang S-Y, McGary EC, Chang S (1989) J. Bacteriol 171:4071-4072

54. Gonzales T, Robert-Baudouy J (1996) FEMS Microbiol. Rev. 18:319-344

55. Taylor A (1993) FASEB J. 7:290-298

56. Taylor A (1993) TIBS 18:167-172

57. Taylor A (ed) (1996) Aminopeptidases. R. G. Landes Co., Austin, TX USA

58. Molecular graphics and analyses were performed with the UCSF Chimera package (http://www.cgl.ucsf.edu/chimera). Chimera is developed by the Resource for Biocomputing, Visualization, and Informatics at the University of California, San Francisco, with support from the National Institutes of Health (National Center for Research Resources grant 2P41RR001081, National Institute of General Medical Sciences grant 9P41GM103311). 


\section{Figure Captions}

Figure 1. Biosynthetic pathways of meso-diaminopimelic acid and lysine in bacteria.

Figure 2. Reaction catalyzed by DapE.

Figure 3. Ribbon diagram of the X-ray crystal structure of the DapE from $H$. influenzae showing the dimer and catalytic structural domains. Zinc ions are labeled in black (Zn1-the catalytic zinc) and grey (Zn2) [58].

Figure 4. Active site of the A) Zn_DapE and B) ZnZnDapE from H. influenzae [58].

Figure 5. Surface rendering of [ZnZn(DapE)] from $H$. influenzae showing the charge distribution and depicting the crescent-shaped active site cavity [58].

Figure 6. Charged residues near the dinuclear active site of DapE that may play a role in substrate recognition and binding process [58].

Figure 7. Proposed catalytic mechanism of DapE.

Figure 8. Structures of L-captopril and L-penicillamine. 


\section{Figure 1}

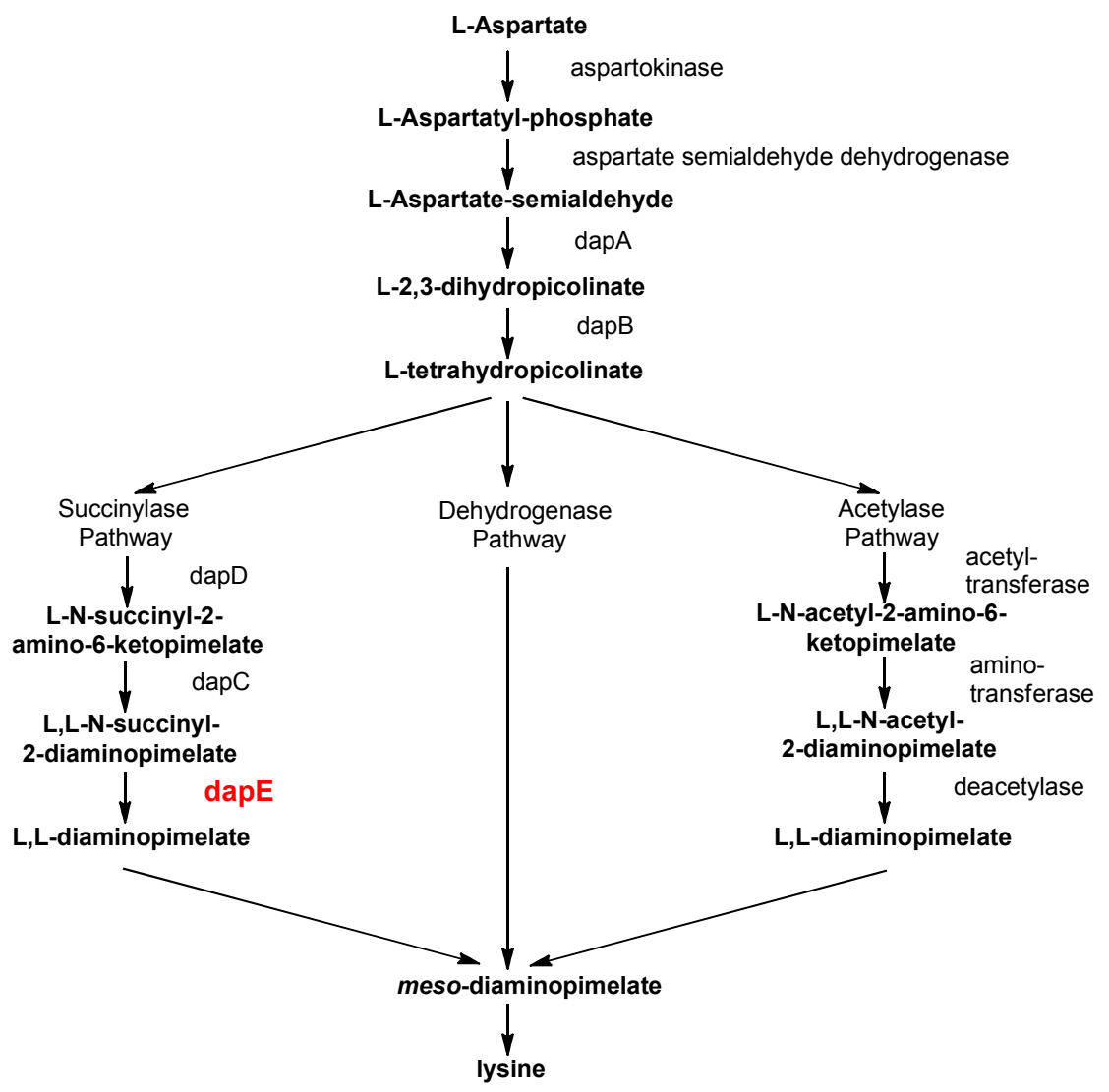


Figure 2

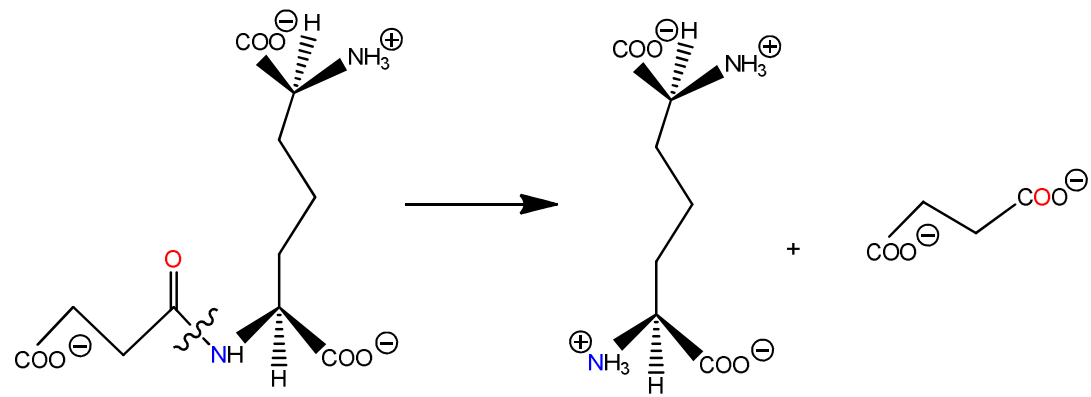

$\mathrm{N}$-succinyl-L,L-diaminopimelic acid (SDAP)

L,L-diaminopimelic acid

Succinic acid 
Figure 3

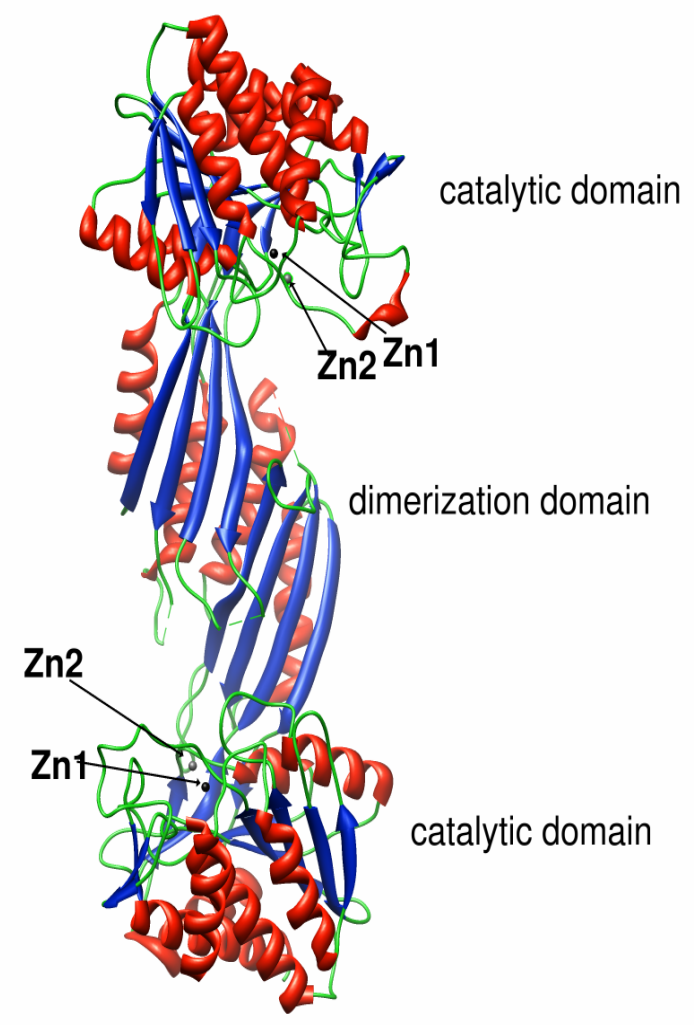


Figure 4

A

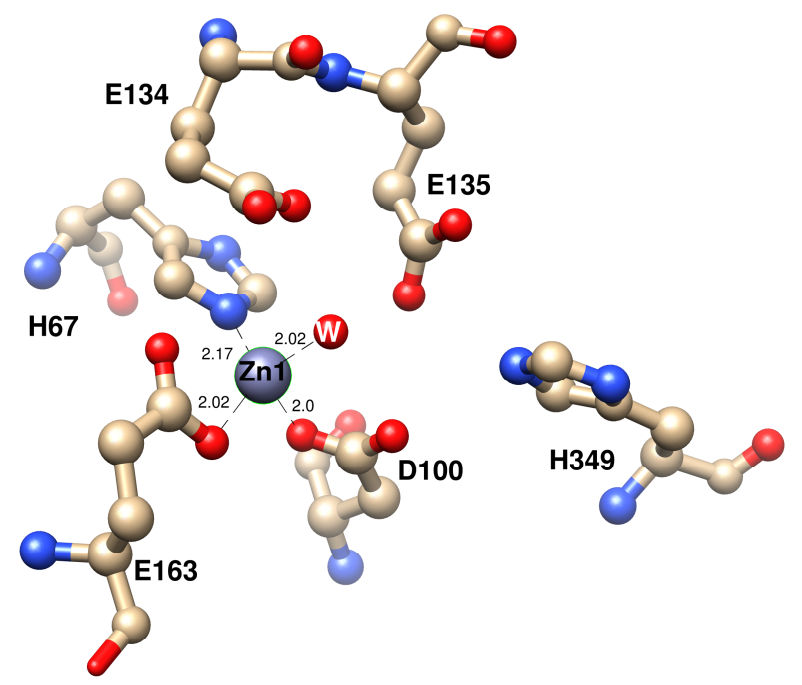

B

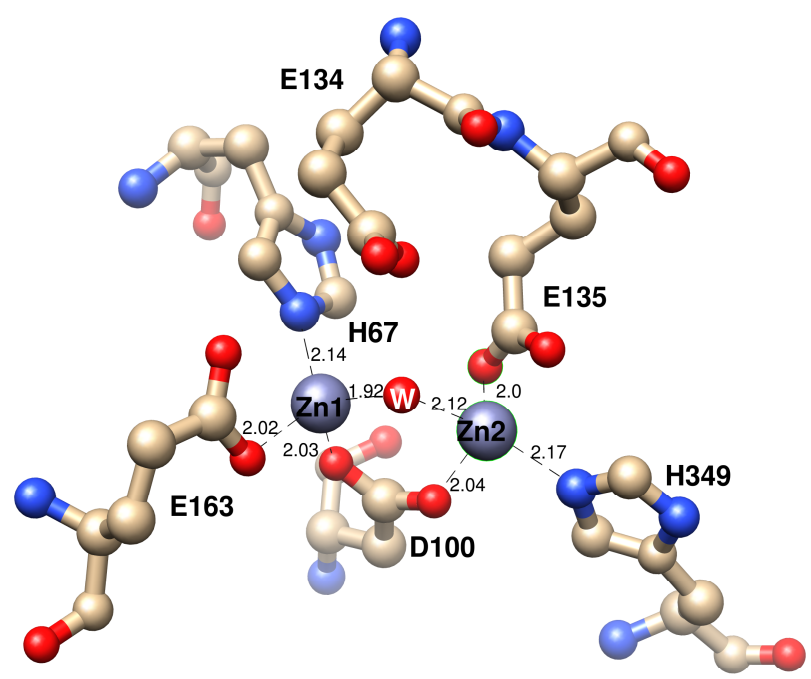


Figure 5

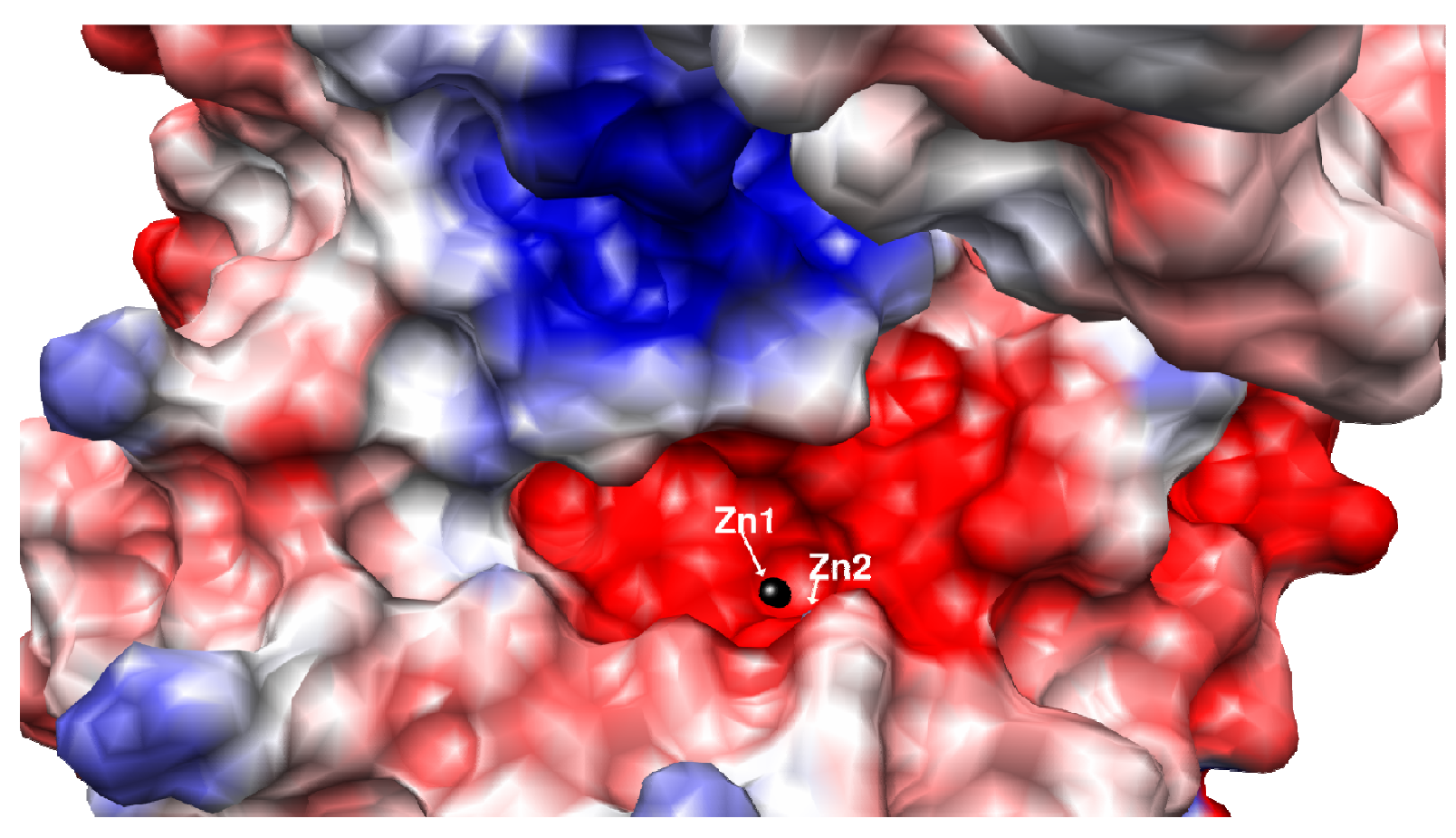


Figure 6

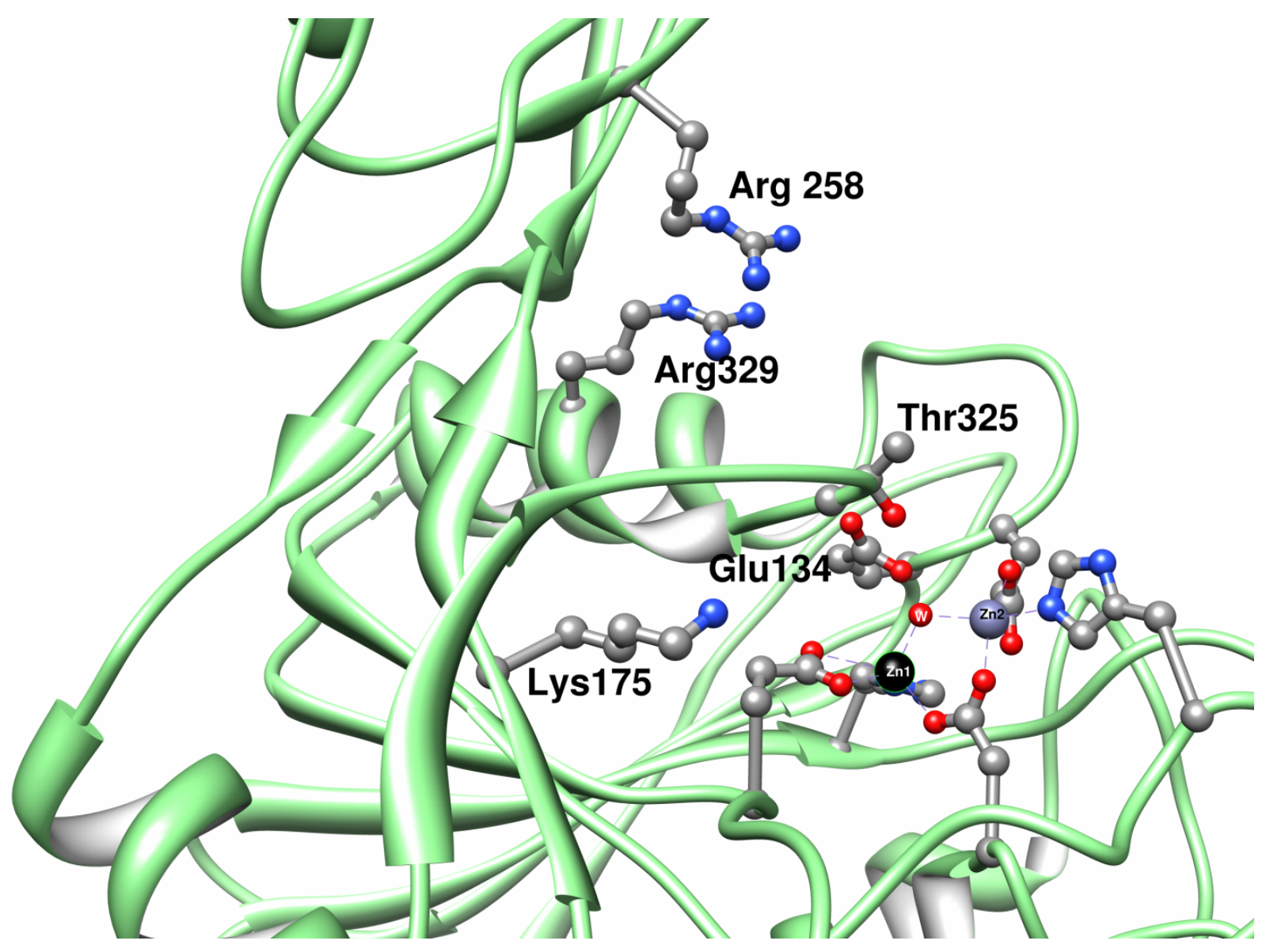


Figure 7

A

A $\quad \overbrace{}^{-\mathrm{Glu}_{134}}$

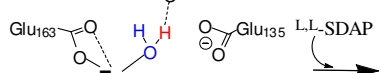

Asp

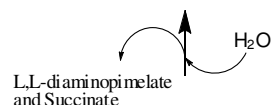

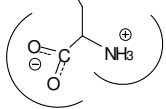

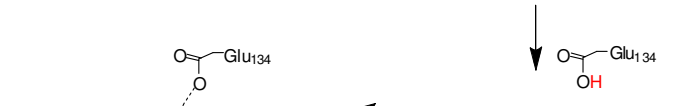

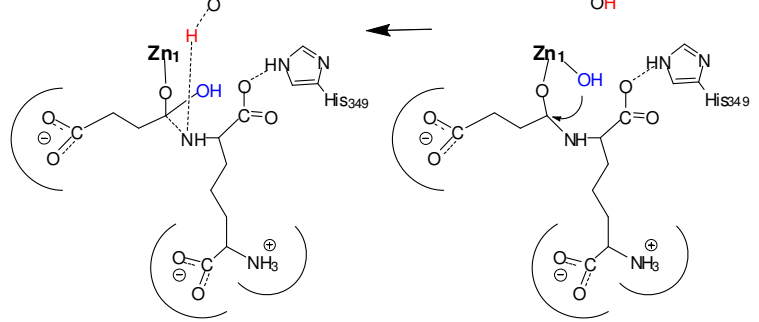

B
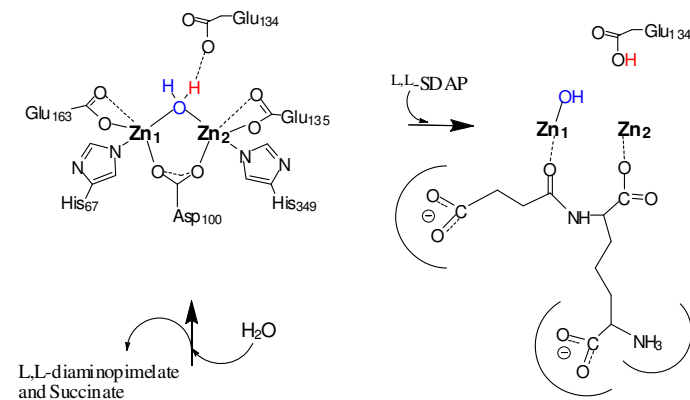

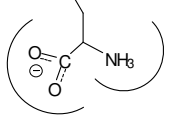

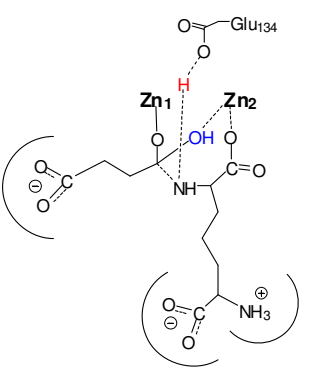

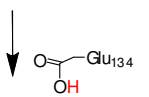

$\longleftarrow$ 
Figure 8
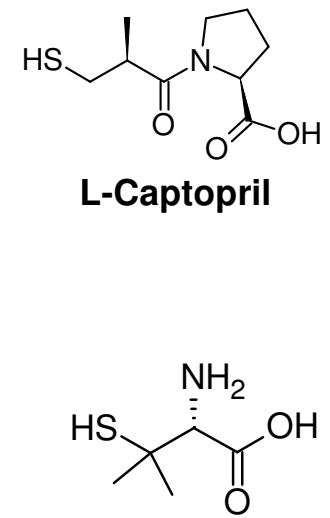

L-Penicillamine 